\title{
DINAMICA VEGETAL DE LA PRADERA-TAMARIZAL EN EL GALACHO DE JUSLIBOL (ZARAGOZA)
}

\author{
Paloma IBARRA, Gustavo BARRON, Luis Alberto LONGARES, \\ Fernando PEREZ y Pedro SESE
}

Departamentó de Geografía y Ordenación del Territorio Universidad de Zaragoza

\begin{abstract}
Resumen: Se establece un diagnóstico sobre la dinámica vegetal de un mosaico de formaciones vegetales denominado "pradera-tamarizal" localizado en el sector NO. del Galacho de Juslibol.
\end{abstract}

Palabras claves: Galacho, pradera-tamarizal, dinámica vegetal, formaciones vegetales, análisis diacrónico.

\begin{abstract}
Abstrats: This paper presents a diagnosis about vegetation dynamics of a mosaic of botanical communities named "prairie-scrub of Tamarix gallica" and placed at the NW sector of the "Galacho de Juslibol" (Valle del Ebro, Zaragoza).

Key words: vegetation dynamics, mosaic of botanical communities, "prairiescrub of Tamarix gallica", diachronic analysis, Valle del Ebro, Zaragoza.
\end{abstract}

\section{LOCALIZACION DEL AREA DE ESTUDIO}

El Galacho de Juslibol es un meandro abandonado del Río Ebro, localizado al NO. de la ciudad de Zaragoza. Dentro de este espacio, el sector objeto de estudio queda ubicado en el extremo NO. del galacho (Fig. 1), a unos 25 metros de la lámina de agua del antiguo lecho. Se trata de un área bastante bien delimitada por una banda de vegetación formada principalmente por Populus alba, Tamarix gallica, Rubus ulmifolius, etc., que la aisla de los campos de cultivo por el NO y de la lámina de agua al SE. 


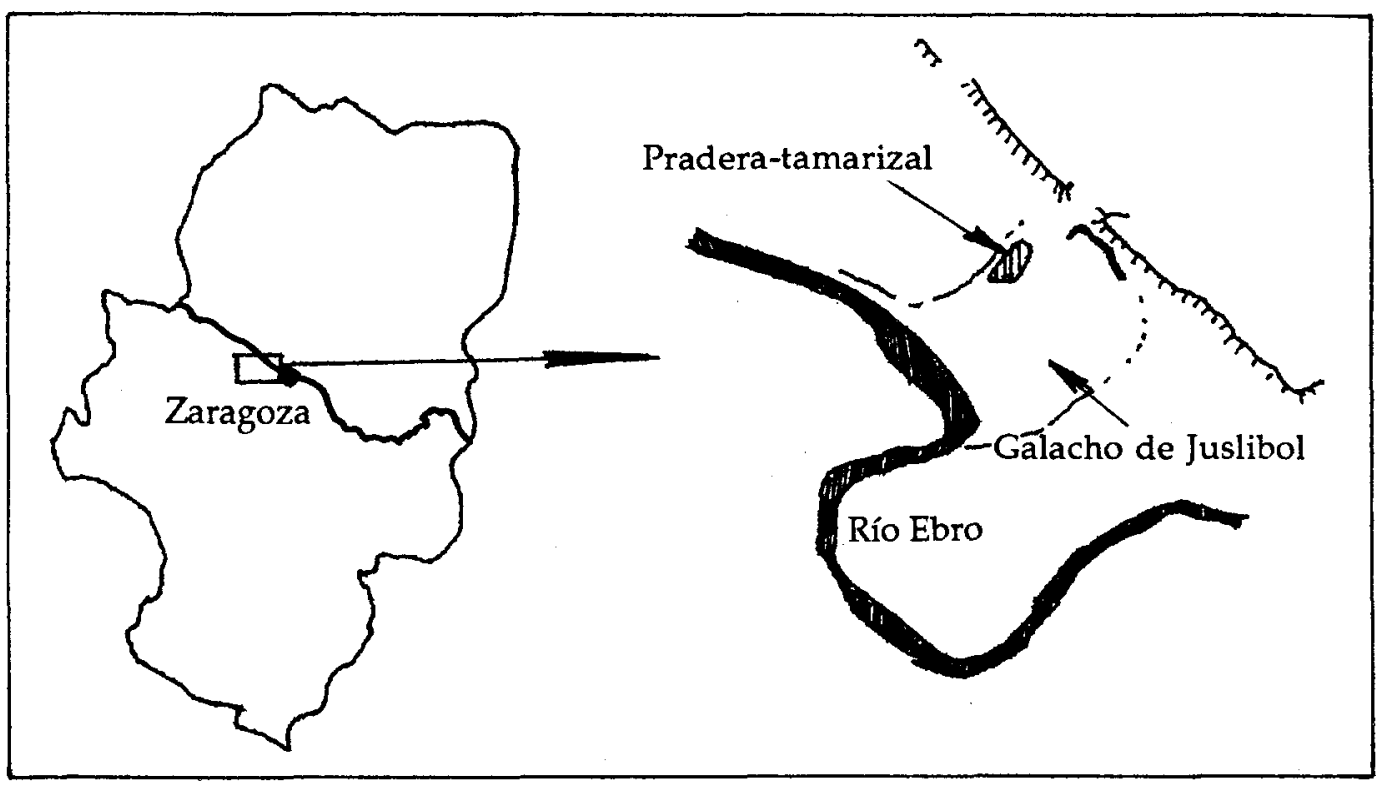

Figura 1.- Localización del área de estudio.

La zona de estudio constituye un mosaico de formaciones vegetales al que se ha denominado "pradera-tamarizal". Las fluctuaciones del nivel freático en relación con la dinámica fluvial del Río Ebro, la extracción de áridos y el uso ganadero al que ha estado sometido este espacio, son algunos de los factores que han condicionado el desarrollo de la pradera-tamarizal.

\section{OBJETIVOS Y METODOLOGIA}

El objetivo planteado es establecer un diagnóstico sobre la dinámica vegetal de la pradera-tamarizal en su conjunto teniendo en cuenta la tendencia evolutiva de las distintas formaciones vegetales que la componen. El proceso metodológico seguido ha sido el siguiente:

- Estudio de la evolución de la vegetación en las últimas décadas de todo el sector de la actual pradera-tamarizal mediante fotografías aéreas. 
- Reconocimiento de todo el sector directamente sobre el terreno y selección de una parcela modelo de unos $500 \mathrm{~m}^{2}$ que aglutinase, y de forma representativa, a todas las formaciones vegetales existentes en el momento actual.

- Estudio detallado de la parcela modelo seleccionada, realizando inventarios de vegetación de cada una de las formaciones vegetales presentes en los que se ha recogido para cada especie el índice de abundancia/dominancia o recubrimiento (Ab/D) de Braun-Blanquet (1979).

- Diagnóstico de la tendencia evolutiva de la vegetación.

\section{ANALISIS DIACRONICO DE LA VEGETACION}

En el año 1957, el área de estudio formaba parte del llamado "Soto de Alfocea". Formaciones vegetales de carácter leñoso arborescente, arbustivo y comunidades de pradera cubrían este soto en un $70 \%$ y $30 \%$ respectivamente.

En 1984 se advierte una gran reducción de la superficie con vegetación espontánea de este Soto de Alfocea, pues queda relegada a una estrecha banda, de aproximadamente $200 \mathrm{~m}$. de anchura, conectada directamente a la lámina de agua del galacho y limitada al Oeste por el camino principal que la separa los campos de cultivo. Esta reducción es debida a las roturaciones de los años 60-70 para la puesta en cultivo de tierras anteriormente marginales.

El área de estudio corresponde a la banda en la que se mantuvo la vegetación. El hecho de que sea una zona susceptible de recibir periódicamente inundaciones, la hace especialmente desfavorable para el uso agrícola y explica que no fuese cultivada. Sin embargo, parece ser que tuvo un aprovechamiento ganadero como zona de pastos siendo especialmente intenso en este intervalo (1960-1984) en el que predominan las especies herbáceas. También ha sido zona de extracción de gravas. En 1984 estaba cubierta en un $60 \%$ por formaciones herbáceas o de "pradera", en un $35 \%$ por áreas de matorral subarbustivo y en un pequeño porcentaje por rodales arborescentes de Tamarix gallica.

El fotograma de 1988 muestra la disminución de las comunidades de pradera que ocupan aproximadamente un $30 \%$ detectándose un incremento del matorral y de las formaciones arborescentes de Tamarix gallica que ahora ocupan el $40 \%$ y $30 \%$ respectivamente. Posiblemente, este aumento de las formaciones leñosas a costa de las herbáceas esté en relación con el cese de la utilización ganadera de este espacio. 
Finalmente, entre 1988 y 1994 la vegetación arborescente y arbustiva ha seguido aumentando progresivamente. Los núcleos de tamarizal han incrementado tanto su superficie como su porte e igualmente el cortejo de especies leñosas subarbustivas que los circundan; ello en detrimento de las formaciones herbáceas que ocupan áreas de dimensiones cada vez más reducidas. Así, en el momento actual se estima que la pradera cubre únicamente el $10-15 \%$, mientras que el matorral y el tamarizal suponen el $40 \%$ y el $35 \%$ respectivamente, lo que da una idea de la evolución que han experimentado las formaciones vegetales de este sector.

\section{ESTUDIO DE LAS DISTINTAS FORMACIONES VEGETALES DE LA PRADERA- TAMARIZAL}

En el conjunto del espacio denominado pradera-tamarizal se pueden identificar seis formaciones vegetales: pradera juncal, pradera "propiamente dicha" o pradera de agramen y cuernecillo, pradera de fondo de pequeñas hondonadas, matorral de regaliz, tarayal o tamarizal y matorral de achicoria y lino.

Con objeto de representar, tanto los principales rasgos de su composición florística y de su estructura, como la ubicación y relaciones de las distintas formaciones, se ha realizado un corte transversal en la parcela modelo seleccionada (Fig. 2). Como puede observarse, tres de las formaciones citadas son herbáceas (pradera), dos son subarbustivas (matorral) y otra es entre arbustiva y arborescente (tamarizal).

La disposición que las diferentes formaciones vegetales presentan a modo de mosaico dentro de este espacio, es lo que justifica la denominación de "praderatamarizal" para el conjunto de la formación vegetal de este sector. Dicha disposición se explica, por un lado, por factores fundamentalmente edáficos: variaciones espaciales de humedad edáfica, distintas concentraciones de cloruros y nitratos, variaciones microtopográficas etc. $y$, por otro lado, la presencia de distintas etapas dentro del proceso evolutivo de la vegetación que corresponde a estos ambientes. A continuación se describen estas seis formaciones vegetales incluyendo en cada caso un inventario florístico representativo.

\section{Pradera juncal}

La pradera-juncal tiene pequeñas dimensiones y se localiza en una zona con gran acumulación de limos, que bordea al carrizal. Es un área que se encharca con facilidad al superar mínimamente el nivel de las aguas del galacho, del cual le separa una 
barrera infranqueable de Salix alba, Rubus ulmifolius, etc. Los juncos constituyen un herbazal denso que se encuentra en buena parte tumbado como consecuencia de las inundaciones periódicas. En la composición florística de esta formación vegetal, como se observa en el inventario que se presenta, destacan especies típicas de prados húmedos y pantanos como Eleocharis palustris y Paspalum paspalodes.

- Eleocharis palustris

- Paspalum paspalodes

- Ecballium elaterium

- Scirpus holoschoenus

- Cyperus longus

- Cynodon dactylon

- Althaea officinalis

- Oenanthe pimpinellifolia

- Lythrum salicaria

\begin{tabular}{c}
$\mathrm{Ab} / \mathrm{D}$. \\
\hline 3 \\
3 \\
2 \\
1 \\
1 \\
1 \\
1 \\
1 \\
1
\end{tabular}

En contacto con esta pradera-juncal se encuentra una pequeña balseta con predominio de aneas.

\section{Pradera o pradera de agramen y cuernecillo}

Los herbazales con dominio de agramen (Cynodon dactylon) y cuernecillo (Lotus tenuis) constituyen el tipo de pradera con mayor extensión en el momento actual, pese a ocupar un porcentaje escaso en el conjunto del sector estudiado. Se corresponden con zonas de menor encharcamiento que las anteriores y posiblemente hayan sido pastoreadas de forma intensa durante bastante tiempo. En el momento actual estas praderas no se pastorean pero si parecen bastante transitadas y pisoteadas. La composición florística actual es testimonio de la utilización antrópica pasada de estas praderas.

\section{$\underline{\mathrm{Ab} / \mathrm{D}}$.}

- Cynodon dactylon $\quad 3$

- Lotus tenuis 3

- Atriplex hastata 2

- Rumex crispus 2

- Althaea officinalis 1

- Carex divisa 1

- Calystegia sepium 1

- Cichorium intybus 1 


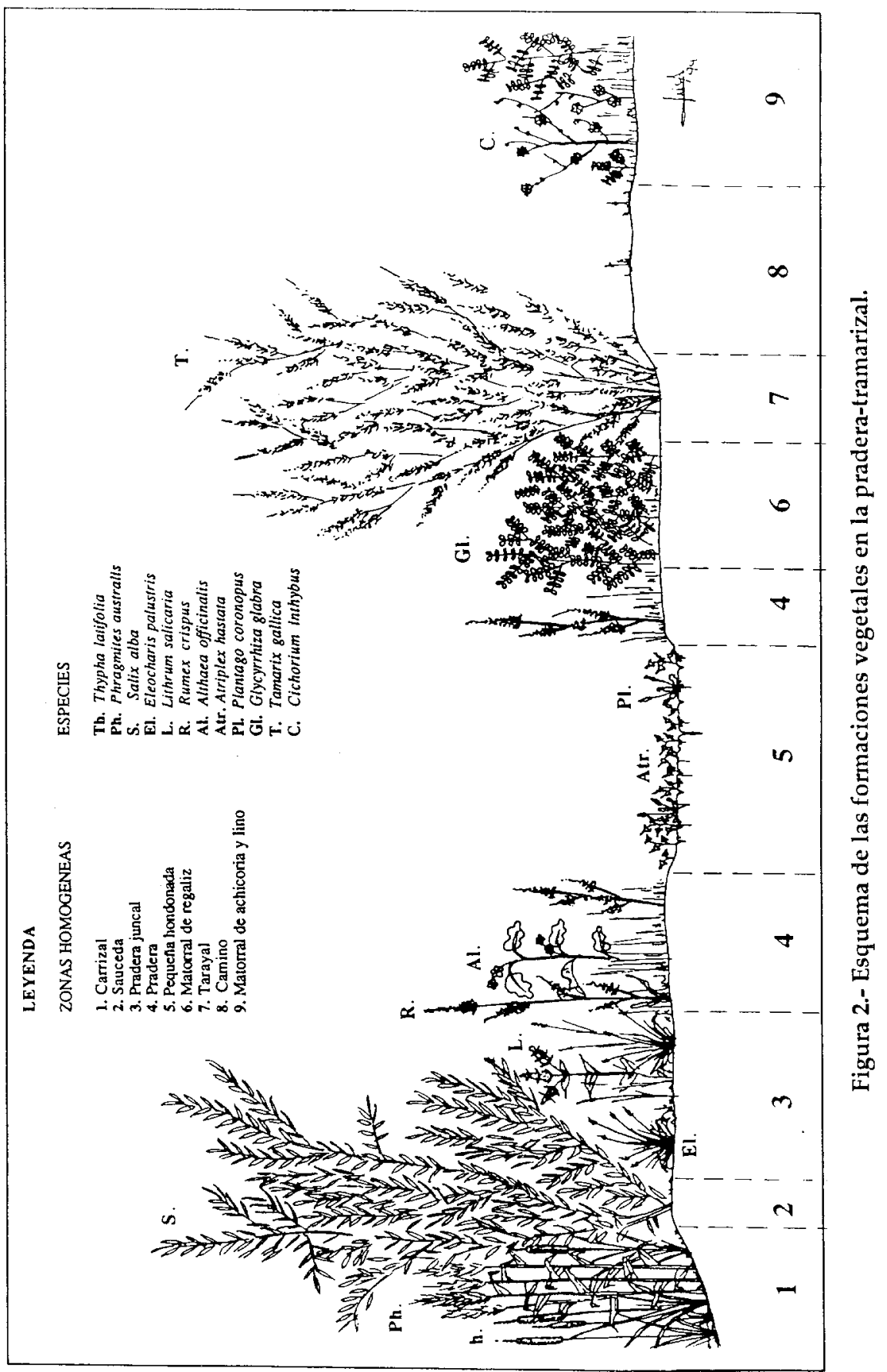


$\mathrm{Ab} / \mathrm{D}$.

- Galium aparine

1

- Scirpus holoschoenus 1

- Conyza sp. 1

- Plantago minor 1

- Eleocharis palustris 1

- Otras gramineas 1

\section{Pradera de fondo de pequeñas hondonadas}

A lo largo de toda la pradera-tamarizal existen variaciones microtopográficas que se traducen en diferencias en la cubierta vegetal. Así, se encuentran pequeñas depresiones $\mathrm{u}$ hondonadas que son más evidentes cuando la elevación del nivel freático provoca su encharcamiento o cuando se encuentran totalmente desecadas y cuarteadas debido a la retracción de las arcillas limosas. En estas depresiones, como se ve en el inventario presentado, el recubrimiento vegetal es muy denso, aunque de porte muy escaso siendo la composición florística muy pobre al dominar casi por completo Plantago coronopus y Atriplex hastata; únicamente, en las márgenes de las hondonadas aparece Agropyron junceum.

$\begin{array}{lc} & \underline{\mathrm{Ab} / \mathrm{D}} . \\ \text { - Atriplex hastata } & 4 \\ \text { - Plantago coronopus } & 4 \\ \text { - Agropyron junceum } & 1\end{array}$

\section{Matorral de regaliz}

Esta formación vegetal subarbustiva está dominada por el regaliz (Glycyrrhiza glabra), especie típica de los tamarizales, acompañada de un cortejo florístico mayoritariamente herbáceo (ver inventario). Es un tipo de matorral que con frecuencia se localiza alrededor de los rodales arborescentes de Tamarix gallica y en las zonas de mayor humectación o salinización del sustrato (Regato, 1988). La composición florística de esta formación es la siguiente:

\section{$\underline{\mathrm{Ab} / \mathrm{D}}$.}

- Glycyrrhiza glabra 4

- Cynodon dactylon 3

- Hordeum hystrix 3 
- Plantago minor 2

- Linum bienne 2

- Medicago sativa 2

- Cichorium intybus 1

- Deschampsia caespitosa 1

- Calystegia sepium +

- Atriplex hastata +

\section{Tarayal o Tamarizal}

En el momento actual, el tamarizal constituye una formación vegetal arborescente en la mayor parte de los casos. Su disposición en el conjunto del sector estudiado es muy característica pues forma pequeños rodales frecuentemente desarrollados sobre elevaciones del terreno y localizados en mayor medida en las áreas limítrofes o banda externa en contacto con otras formaciones arbóreas.

Existe una cierta diversidad en la estructura y composición florística de los rodales de tamariz debida a posibles diferencias de pastoreo en épocas pasadas, a la propia localización más próxima o lejana a las formaciones arbóreas de la periferia o a las herbáceas de la zona central y/o próxima al galacho y a pequeñas variaciones edáficas y topográficas. Un ejemplo de rodal de tamariz bastante denso y próximo al borde externo es el del inventario incluido; en otros casos no existe apenas estrato arbustivo, siendo más abundantes las herbáceas.

\section{$\mathrm{Ab} / \mathrm{D}$.}

- Tamarix gallica 4

- Cynodon dactylon 3

- Althaea officinalis 2

- Rubus ulmifolius 2

- Calystegia sepium 1

- Oenanthe pimpinellifolia +

- Salix alba +

- Crataegus monogyna +

- Ulmus minor +

- Rubia peregrina + 


\section{Matorral de achicoria y lino}

Este tipo de matorral subarbustivo está dominado por la achicoria (Cichorium intybus) y el lino (Linum bienne) y se localiza en el extremo NO de la praderatamarizal. Aparece separado de las formaciones anteriores por el camino que une la mota con el camino principal que atraviesa el galacho y que genera un pequeño salto topográfico que explica la menor humectación del suelo que caracteriza a este sector (Fig. 2). En consecuencia, las especies que componen este matorral están adaptadas a condiciones algo más xéricas que las del otro matorral descrito aunque hay algunas comunes como puede observarse en el inventario siguiente.

\section{$\underline{\mathrm{Ab} / \mathrm{D}}$.}

- Cichorium intybus 4

- Linum bienne 4

- Glycyrrhiza glabra 2

- Avena sterilis 2

- Rumex crispus 1

- Tamarix gallica 1

- Plantago minor 1

- Poa pratensis 1

- Calystegia sepium 1

- Althaea officinalis 1

- Ulmus minor +

- Hordeum histrix + +

\section{DIAGNOSTICO SOBRE LA DINAMICA DE LA VEGETACION}

Para establecer el diagnóstico de la dinámica de la vegetación se ha tenido en cuenta tanto el análisis diacrónico de las principales formaciones vegetales a través de fotografía aérea, como el estudio en detalle de las diferentes comunidades existentes sobre el terreno.

Lo más interesante del análisis diacrónico realizado es comprobar como en los años 50 (vuelo 1956) el sector estudiado correspondía a un tamarizal con algunas áreas de matorral y únicamente una pequeña superficie era pradera. Desde este momento al del siguiente fotograma de 1984, se constata una evolución claramente regresiva de la vegetación puesto que se hace dominante la pradera, evidenciando un fuerte retroceso del tamarizal y del matorral. Esta regresión es debida, posiblemente, a una 
explotación antrópica más intensa de espacio que, concretamente debió ser utilizado como pasto paralelamente a la puesta en cultivo del sector más NO del antiguo Soto de Alfocea, así como lugar de extracción de áridos hasta época reciente.

Esta tendencia regresiva de la vegetación se invierte completamente en el transcurso de la última década (desde 1984 hasta 1994) puesto que en la actualidad la pradera ha experimentado un fuerte retroceso permitiendo el avance del tamarizal y del matorral; estas formaciones dejan de localizarse fundamentalmente en las márgenes para adentrarse en el espacio ocupado con anterioridad por la pradera, configurando así el actual mosaico de formaciones vegetales. Parece claro que en los últimos años, la tendencia evolutiva de la vegetación ha sido progresiva evolucionando hacia un tamarizal.

Descendiendo al detalle que aporta el trabajo de campo y el reconocimiento de cada una de las formaciones vegetales existentes, se puede confirmar este primer diagnóstico y concretar cuál es la secuencia evolutiva o relaciones dinámicas entre estas comunidades que se presentan en mosaico en el momento actual.

Este mosaico de formaciones vegetales queda reflejado en la Figura 2 y, a grandes rasgos se diferencian dos zonas:

- Una zona muy cercana a la lámina de agua del galacho en la que, junto al carrizal y a la sauceda, se localiza la pradera juncal respondiendo a unas condiciones de mayor humedad y de microtopografía poco acusada que facilitan su encharcamiento esporádico.

- Una segunda zona mucho más amplia y más alejada de la lámina de agua en la que se localizan el resto de las formaciones vegetales y que presenta pequeñas variaciones topográficas que condicionan, en parte, la vegetación. Así en las pequeñas hondonadas hay una cubierta de Plantago coronopus y Atriplex hastata, junto a ellas se encuentra la pradera de agramen y cuernecillo, ambas parecen haber estado sometidas a mayor presión (pastoreo y pisoteo). La pradera entra en contacto, en el sector al Este del camino, con el matorral de regaliz que, a su vez circunda los rodales de tamarizal que se sitúan en los enclaves topográficos algo más elevados. Al Oeste del camino en el sector más elevado topográficamente y de menor humedad edáfica el matorral que se desarrolla entre la pradera y el tamarizal es el matorral de achicoria y lino.

Las relaciones dinámicas que pueden establecerse entre estas formaciones vegetales, que representan distintas fases sucesionales de los bosques riparios (Rubieto-Populetum), pueden esquematizarse de la forma que se recoge en el esquema anexo. 
Como se observa, se plantea para la zona más húmeda de la pradera juncal la evolución hacia una sauceda de orla, como aquélla con la que contacta o hacia una pradera; ello dependerá fundamentalmente de la estabilidad, ascenso o descenso del nivel freático que favorecerá la evolución en una u otra dirección. En todo caso, mientras se mantengan las condiciones actuales la tendencia de esta pradera juncal es bastante estable.

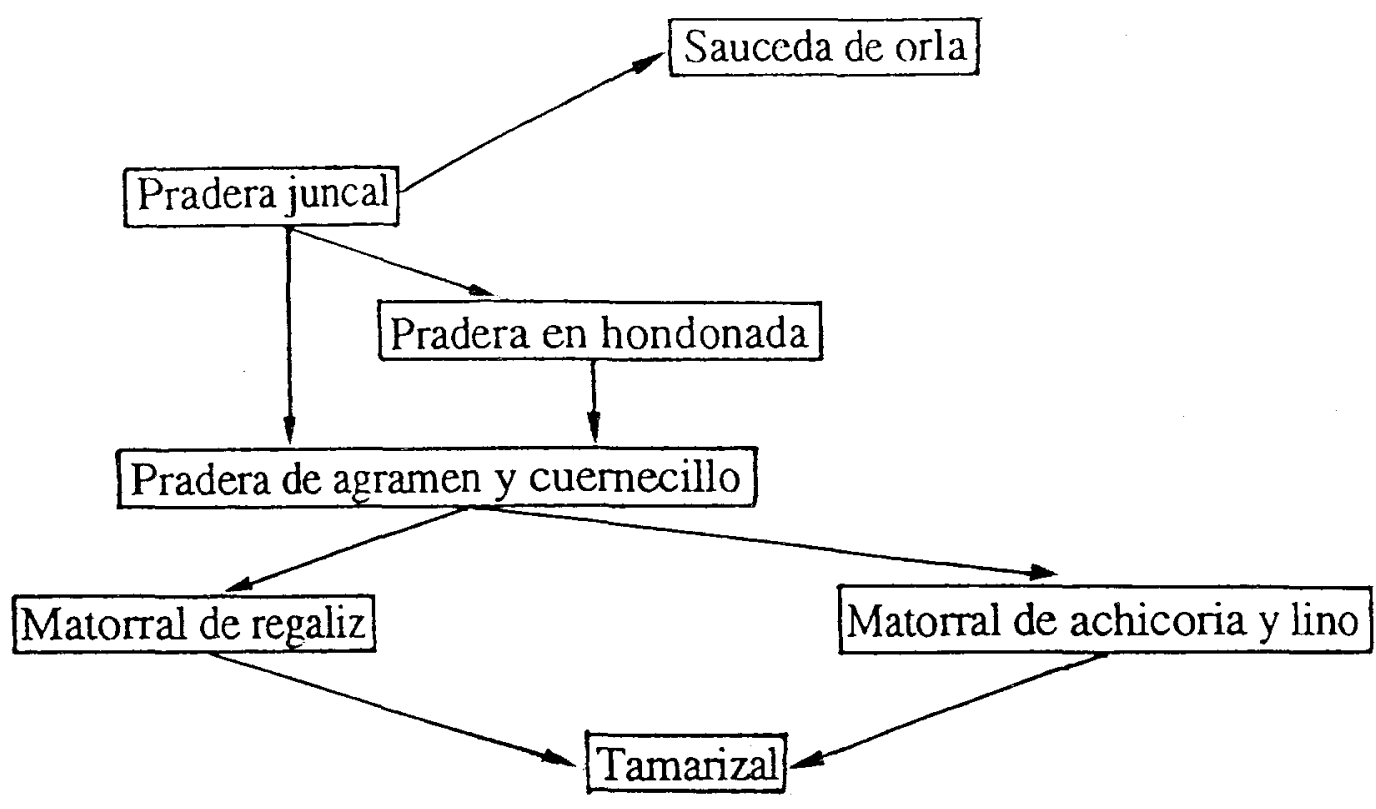

Desde esta pradera-juncal se puede evolucionar, en relación al descenso del nivel freático y a una cierta presión antrópica y ganadera (Regato, 1988), hacia formaciones de pradera que pueden pasar por las propias de los fondos de hondonadas algo más humectadas y degradadas o evolucionar directamente a praderas de agramen y cuernecillo. A partir de estas situaciones se alcanzan las etapas de matorral que pueden estar dominados por el regaliz cuando el suelo es algo más húmedo y por la achicoria y el lino cuando el nivel freático es algo más bajo. Son éstas las etapas más próximas al tamarizal que con sus pequeñas variaciones constituye la etapa madura de la sucesión vegetal en un espacio de estas características, sin descartar la posibilidad a largo plazo y en relación con la evolución del freático y de la salinidad, de una evolución hacia una chopera. 
En definitiva, el espacio de la pradera-tamarizal evoluciona hacia un tamarizal, con la posible excepción de la pradera-juncal que puede permanecer a corto-medio plazo estable y evolucionar posteriormente hacia una sauceda o, igualmente, hacia un tamarizal. Esta secuencia evolutiva coincide básicamente con la señalada por Regato (1988) para el Galacho de la Alfranca aunque en nuestro caso se observa una mayor variedad en los tipos de pradera y de matorral.

Finalmente, es conveniente destacar que estos medios son poco homogéneos presentando variaciones espaciales (edáficas, topográficas) y temporales (inundaciones) importantes que provocan tanto la variabilidad de comunidades vegetales como la de las tendencias evolutivas.

Este artículo se inscribe en el apartado de Análisis de la vegetación del Estudio de Recuperación del Galacho de Juslibol. Fase II. realizado por la Univ. de Zaragoza y financiado por el Exmo. Ayuntamiento de Zaragoza.

\section{BIBLIOGRAFIA}

REGATO, P. (1988): "Contribución al estudio de la flora y la vegetación del Galacho de la Alfranca en relación con la evolución del sistema fluvial". Naturaleza en Aragón 3. Diputación General de Aragón.

BRAUN BLANQUET, J, (1979): Fitosociología. Ed. Blume.

UNIVERSIDAD DE ZARAGOZA (1990): Estudio de recuperación del Galacho de Juslibol. Fase I. Ayuntamiento de Zaragoza.

UNIVERSIDAD DE ZARAGOZA (1994): Estudio de recuperación del Galacho de Juslibol. Fase II. Ayuntamiento de Zaragoza.

Fotografía aérea escala 1:33.000, año 1957. Servicio Geográfico del Ejército.

Fotografía aérea escala 1:30.000, año 1984. CECAF.

Ortofoto escala 1:5.000, año 1988. Centro de Gestión Catastral y Cooperación Tributaria. $\mathbf{M}^{\circ}$ Economía y Hacienda. 